\title{
The use of human-in-the-loop and constructive simulation to support operations research into $\mathrm{MH}-60 \mathrm{R}$ tactics development
}

\author{
$\underline{\text { Arvind Chandran }}^{\text {a, }}$ Nik Luketić a, Christopher Stewart ${ }^{\text {a }}$ and Mina Shokr ${ }^{\text {a }}$ \\ a Defence Science and Technology Group, Fishermans Bend, Victoria, AUSTRALIA \\ E-mail: Arvind.Chandran@dsto.defence.gov.au
}

\begin{abstract}
Joint and Operations Analysis Division (JOAD) of Defence Science and Technology Group (DST Group) is currently tasked by the Royal Australian Navy (RAN) to provide operations research (OR) support to tactics development for the MH-60R 'Romeo' maritime combat helicopter in the Australian context. This is part of the Romeo acquisition and introduction into service process, as it is integrated into Australia's force-in-being.
\end{abstract}

JOAD employs a number of OR techniques of varying fidelity to support tactics development, with the selection of methods depending on the nature and complexity of the requirement. One of the approaches employed extensively is the complementary use of human-in-the-loop (HIL) and constructive simulation. Several studies have been conducted using this approach to examine and compare the effectiveness of various Romeo tactics over a range of operational environments and requirements.

Accurate elicitation of tacit operator knowledge is known to be a difficult problem. The DST Groupdeveloped Synthetic Human-in-the-loop Air 7000/9000 Research Program (SHARP) provides a framework in which to expose operators to an interactive real-time simulated mission. SHARP provides an immersive environment in which the operator can explore various tactical employment options in real time. Information can be captured describing critical decision points and the operators' decision-making processes during the mission. This information then forms a baseline for first modelling, then simulating, operator and other human behaviours within the context of the mission using a constructive simulation framework. This approach can be utilised to explore existing defence capabilities or new technologies and concepts. The DST Group-developed Combined Helicopter OPerational and Performance Analysis (CHOPPA) constructive simulation framework supports Monte-Carlo simulation and analysis of complex mission scenarios at timescales that are orders of magnitude faster than real-time. Friendly, neutral and enemy platforms, weapons, sensors, communications effects, human behaviours and the operating environment are all mathematically modelled in CHOPPA at varying levels of fidelity. The results of these constructive simulations facilitate a robust statistical analysis to inform tactics development for the RAN.

By using these tools to examine operational issues for the Romeo, DST Group is directly contributing to improving operational effectiveness, enhancing Australia's military capability and ensuring value for money for the RAN.

This paper presents a summary of the SHARP and CHOPPA simulations, their complementary use and the type of study outcomes produced in an example study exploring Romeo tactics development.

Keywords: Human-in-the-loop simulation, constructive simulation, modelling, operations research, elicitation 
Chandran et al., The use of human-in-the-loop and constructive simulation to support operations research into $\mathrm{MH}-60 \mathrm{R}$ tactics development

\section{INTRODUCTION}

The Defence Science and Technology Group (DST Group) employs a number of operations research (OR) techniques of varying fidelity such as spreadsheet analysis, complex simulation or war-gamming to support acquisition and tactics development, with the selection of methods depending on the nature and complexity of the requirement. One of the approaches employed extensively for tactics and concept of operations (CONOPS) development is the complementary use of human-in-the-loop (HIL) and constructive simulation. This paper discusses the background of the two capabilities in the Joint and Operations Analysis Division (JOAD), how they have evolved and, through an example study, how they are jointly used today.

\subsection{Human-in-the-loop (HIL) Simulation}

During the early 2000's, JOAD was providing OR support to the upgrade of the AP-3C sensor suite. Initially, constructive simulation was used to determine the effectiveness of baseline AP-3C sensor tactics. However, it became apparent that new tactics were required to improve the effectiveness of the upgraded sensor suite. As a result, an interactive version of the constructive simulation was developed, and a human crew of AP-3C operators replaced the computational operator models that had previously been developed for the constructive simulation (Bennett et al. 2001).

Following this initial capability, JOAD has developed and employed other human-in-the-loop capabilities for a number of projects to elicit tacit operator knowledge (Iob et al, 2004). One such capability is the DST Group-developed Synthetic Human-in-the-loop Air 7000/9000 Research Program (SHARP), which provides a framework in which operators can be exposed to an interactive real-time simulated mission. SHARP provides an immersive environment in which the operator can explore various tactical employment options in real time. Through after-action reviews, critical decision points and the operators' decision-making processes during the mission are captured, which provides a deeper insight into operator behaviour.

\subsection{Constructive Simulation}

JOAD has been employing constructive simulation approaches for a number of years to provide robust statistical analysis and advice to acquisition and tactics development questions. These different capabilities have supported projects such as the F/A-18 Hornet upgrade (Tidhar et al, 1998), Airborne Early Warning and Control tender evaluation and Armed Reconnaissance Helicopter acquisition (Chandran et al 2007).

In 2010, JOAD commenced development of the Combined Helicopter OPerational and Performance Analysis (CHOPPA) constructive simulation framework. This capability supports Monte-Carlo simulation and analysis of complex mission scenarios at timescales that are orders of magnitude faster than real-time. Friendly, neutral and enemy platforms, weapons, sensors, communications effects, human behaviours and the operating environment are all mathematically modelled in CHOPPA at varying levels of complexity. The results of these constructive simulations facilitate a robust statistical analysis to inform tactics development for the RAN.

\section{COMPLEMENTARY USE OF SHARP AND CHOPPA}

Accurate elicitation of tacit operator knowledge is known to be a challenging problem (Cullen \& Bryman 1988). SHARP is used as a tool to conduct scoping and parameter identification, employing subject matter experts (SMEs) over the course of multiple simulation experiments. This establishes a process to explore military tactics with human input during run time. Analysts then capture both the qualitative (tactics and CONOPS) and quantitative (Measures of Performance (MOPs)) data in SHARP. This captured information then forms a baseline for first modelling, then simulating, operator and other human behaviours as intelligent agents within the context of the mission using a constructive simulation framework (Macal \& North 2010).

CHOPPA does not simulate the mission in real-time. Instead, the simulated mission including the operator decision making processes is executed at speeds of at least one-thousand times faster than a real-time SHARP trial. This allows the analyst to conduct millions of runs and collect statistically significant conclusions about the mission effectiveness of tactics and platform capabilities (Burton \& Obel 2011). Whereas SHARP focuses on the collection of data such as decision points, decision-making processes and MOPs, CHOPPA collects data to calculate measures of effectiveness (MOEs) of the decisions made by the operator. These MOEs are determined, through consultation between the stakeholders and the analyst, to be the key criteria to assess the impact of different capability and employment options. This information is used to answer the questions of how effectively the tactic, operation or mission was executed. 
Chandran et al., The use of human-in-the-loop and constructive simulation to support operations research into MH-60R tactics development

The flow of information between SHARP and CHOPPA is shown in Figure 1. It should be noted that following the provision of advice to the RAN, the RAN may trial a particular tactic operationally, and return with feedback or updated data to be incorporated into the HIL and/or constructive simulation capabilities. If this occurs, the modelling is refined and the study is updated for further operational testing. This is known as the CONOPS tactics development life-cycle (Figure 2) (Chandran, et al 2007).

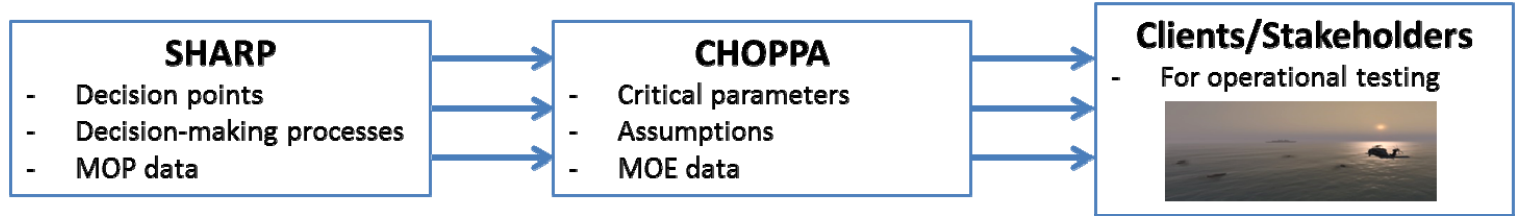

Figure 1. Information Flow between SHARP and CHOPPA.

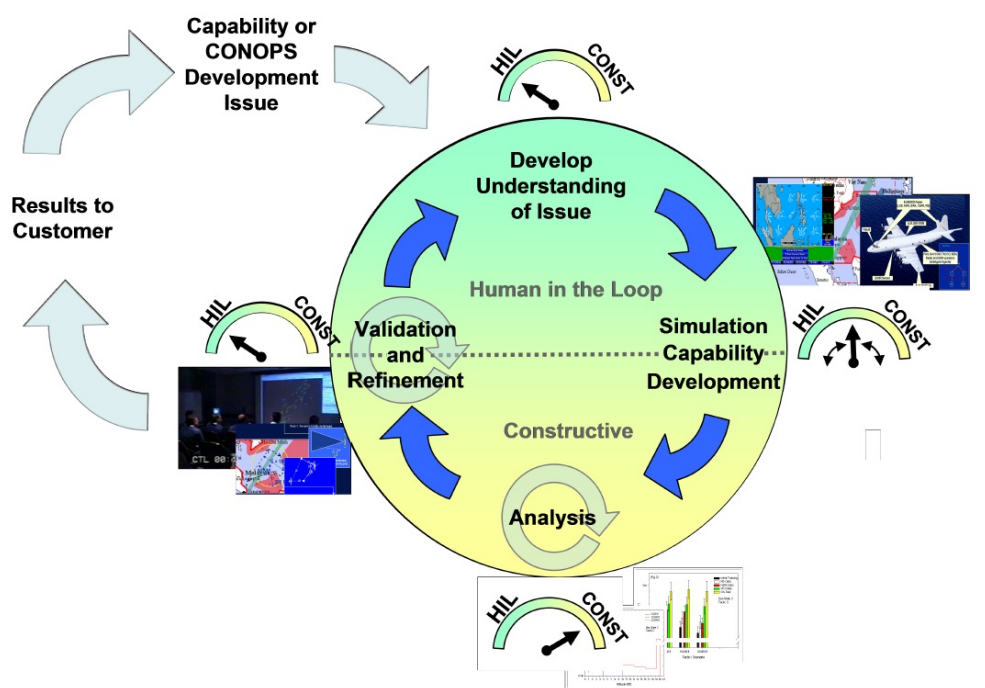

Figure 2. The CONOPS and Tactics Development Lifecycle.

\section{COMPONENTS OF SHARP/CHOPPA}

In order to examine a military operational issue effectively, it is critical that the certain required components of both SHARP and CHOPPA are available and fit-for-purpose. Table 1 describes the key components of the two capabilities that are required for their effective complementary use.

Table 1. The Components of SHARP and CHOPPA

\begin{tabular}{|c|c|c|}
\hline & \multicolumn{2}{|c|}{ ANALYTICAL METHOD } \\
\hline COMPONENT & SHARP & CHOPPA \\
\hline Problem definition & \multicolumn{2}{|c|}{$\begin{array}{c}\text { This should be common across the two capabilities. SHARP is used so that operators can explore the } \\
\text { problem space, while CHOPPA is used for more detailed and robust 'what-if' analysis }\end{array}$} \\
\hline Scenario & \multicolumn{2}{|c|}{$\begin{array}{l}\text { This should be common across the two capabilities, so that the execution and outputs of SHARP and } \\
\text { CHOPPA are aligned and defensible }\end{array}$} \\
\hline Vignette & $\begin{array}{l}\text { Explores a subset of the parameter space in the } \\
\text { context of the scenario }\end{array}$ & $\begin{array}{l}\text { Explores an extended parameter space in the } \\
\text { context of the scenario }\end{array}$ \\
\hline $\begin{array}{l}\text { Modelling, Data and } \\
\text { Assumptions }\end{array}$ & $\begin{array}{l}\text { The key outputs are operator reasoning processes, so } \\
\text { physical modelling can be simplified providing it } \\
\text { does not compromise operator behaviour }\end{array}$ & $\begin{array}{l}\text { Operator behaviour modelling is based on } \\
\text { the observed behaviours in SHARP. } \\
\text { Physical models may need to be higher } \\
\text { fidelity so that study outcomes are valid }\end{array}$ \\
\hline Outputs required & $\begin{array}{l}\text { Decision points, decision-making processes and } \\
\text { MOPs }\end{array}$ & MOEs and their impact on the stakeholder \\
\hline $\begin{array}{l}\text { Information collection/post- } \\
\text { processing }\end{array}$ & Captured by SHARP and observing analysts & $\begin{array}{l}\text { Captured by CHOPPA and post-processed } \\
\text { by analyst/programmers }\end{array}$ \\
\hline Transmissions of outputs & $\begin{array}{c}\text { Decision points and decision-making processes } \\
\text { documented and used in CHOPPA. Outcomes of trial } \\
\text { reported to stakeholders }\end{array}$ & $\begin{array}{l}\text { Key findings and impact and advice from } \\
\text { MOE analysis reported to stakeholders }\end{array}$ \\
\hline
\end{tabular}


Chandran et al., The use of human-in-the-loop and constructive simulation to support operations research into MH-60R tactics development

\section{EXAMPLE STUDY}

Several studies have been conducted using this approach to examine and compare the effectiveness of various MH-60R 'Romeo' tactics over a range of operational environments and requirements. One such study that has been identified examines the effectiveness of the Romeo as an early-warning asset to support an Australian naval frigate (FFH) against fast-boat threats (Homeland Security 2003).

\subsection{Problem Definition}

The objectives of this study were to

i. develop an understanding of the key drivers that influence the Romeo's ability to effectively provide early-warning support to an FFH against fast-boat threats

ii. offer advice and recommendations to Romeo operators on courses of action to improve the Romeo's effectiveness in providing early-warning support to an FFH against fast-boat threats.

The first objective is explored using SHARP. The second objective is analysed using CHOPPA.

The key MOEs that are examined in the overall study are:

- The mean distance from the FFH at which fast-boats are classified by the Romeo

- The $\%$ of unclassified fast-boats that penetrate and enter the FFH vital area $^{i}$ (VA)

\subsection{Scenario}

An FFH and her embarked Romeo are conducting a transit through a heavily populated strait. The strait contains a number of different vessels, such as cargo ships, oil tankers, and pleasure craft. Once airborne, the Romeo will begin its task of establishing the surface picture and notifying the FFH of any suspect vessels. During the transit, adversary forces in the form of a coordinated fast-boat attack may threaten the FFH. As soon as these vessels are identified as hostile, the Romeo will be tasked to inform the FFH of the imminent threat. A schematic of this scenario is shown in Figure 3.

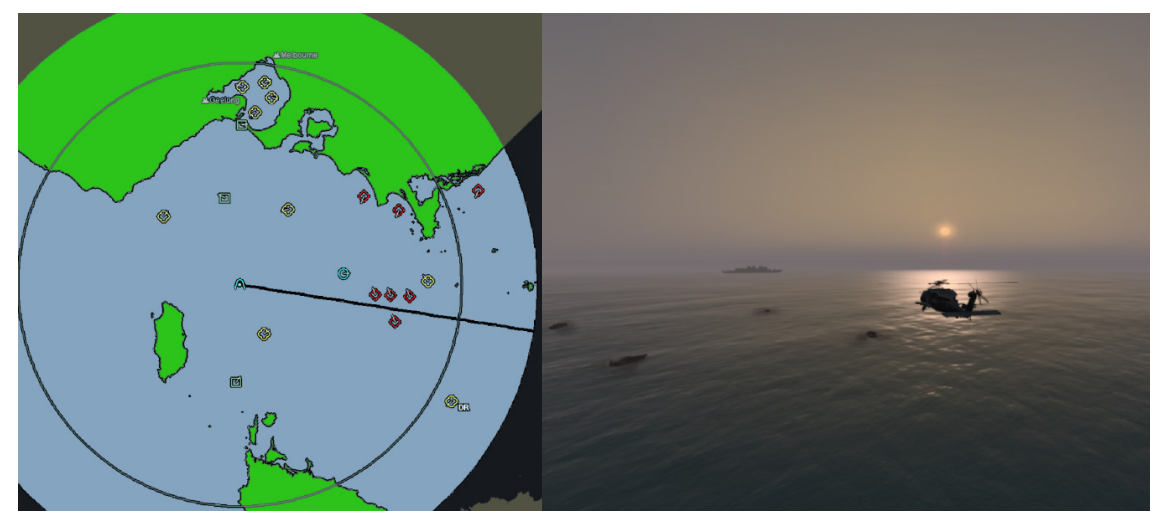

Figure 3. An FFH and Romeo conducting a transit through a strait.

\subsection{Models, Data and Assumptions}

In order to accurately represent the systems that are employed in a scenario such as this, it is important to understand not only which capabilities and functions of the systems need to be represented, but also how the systems interact. The models used in the scenario are determined through consultation with the stakeholders, and an understanding of their capabilities is obtained through discussion with SMEs and reading relevant manuals and documents.

For this example study, a number of models have been used to represent entities in the scenario. Entities such as the cargo ships, oil tankers, pleasure craft, fast-boat threats and the FFH have been modelled to varying levels of platform motion and behavioural complexity, as with other simulation frameworks (TTCP). For this scenario in SHARP, the entities can be controlled in speed and heading by the operator or programed to behave autonomously to represent how they would operate proactively or reactively during such a scenario. When used in CHOPPA, cargo ships, oil tankers and pleasure craft consist of a simple manoeuvre which allows them to travel in a straight line at a constant speed. However, for fast-boat threats and the FFH, 
Chandran et al., The use of human-in-the-loop and constructive simulation to support operations research into MH-60R tactics development

the control of the operator is represented by an intelligent agent, which acts and reacts based on SHARP observations and tactical documentation. In addition, each of the surface vessels is represented by its visual, electronic and acoustic signatures.

The Romeo is represented at a considerably higher level of complexity than the other entities. For this scenario, it consists of a number of sensor, aerodynamic, weapons, counter-measures and communications models which cover its various capabilities. These models are updated by changes in the environment. As with the surface vessels, the Romeo crew, which consists of a pilot, an aviation warfare officer (AvWO) who is responsible for the tactical decision making and aircraft employment and sensor operator (SENSO) who is responsible for sensor employment. Their roles will be represented by operator agents in CHOPPA. Figure 4 shows some of the Romeo systems that are modelled for this study.

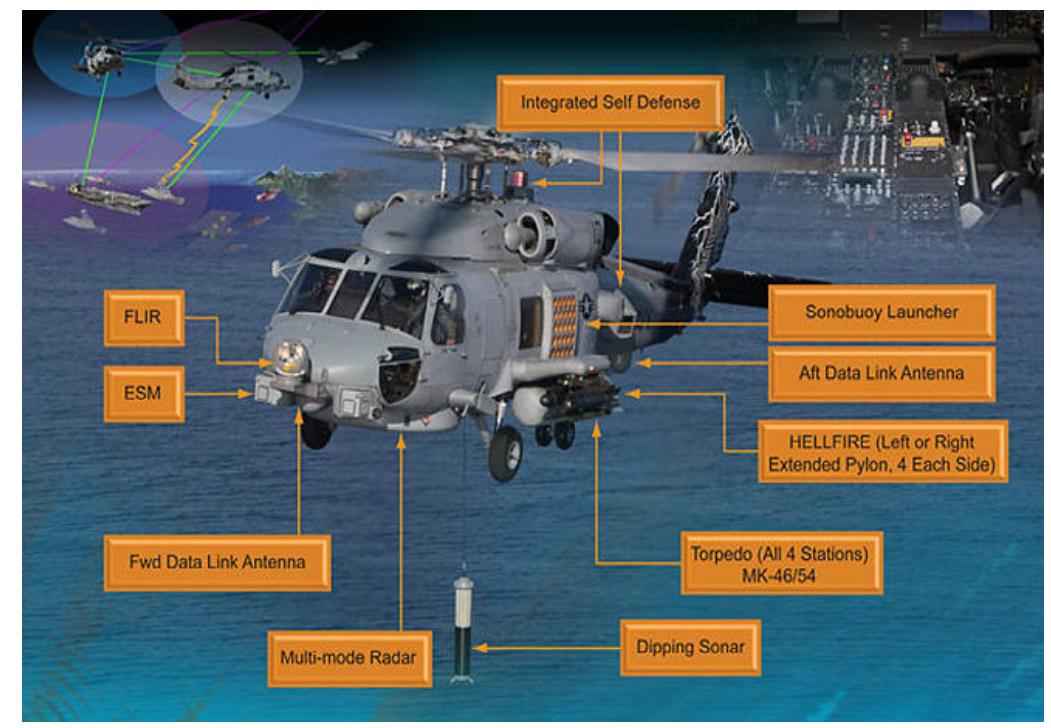

Figure 4. Romeo systems modelled for this study. (http://www.hsm73.navy.mil/sh60b/imgD.jpg)

Accurate system data is required to ensure that the models function as they are intended. Much of the Romeo data used in this study is sourced from technical or flight manuals, whereas some Romeo system data and surface vessel data has been obtained from SMEs in Defence ${ }^{\mathrm{ii}}$.

A number of assumptions must be made to capture the constraints or limitations of the models and data. The validity of these assumptions is discussed with the stakeholder during model development and data acquisition. For this study, assumptions include the estimation of sensor detection ranges against different vessels, as well as the stores configuration (weapons and fuel) for the Romeo.

\subsection{Outputs required}

During a SHARP trial, the pilot, AvWO, SENSO will aim to establish a surface picture and inform the FFH of any hostile threats. The reasoning of 'when', 'why' and 'how' this information is obtained and transferred is captured by analysts through trial observation and post-trial after action review, interviews and discussion. In addition, the crew members are asked about mechanisms that would be used to improve their effectiveness in their roles, and this information is also recorded and documented.

Given the small number of vignettes, typically less than 10, the quantitative outputs that are derived from the trial are used as a rough guide for how the MOEs from a more detailed analysis may look.

Following consultation with participants and documentation of outputs from SHARP, key parameters are identified that would have an impact on measuring the success of the missions. For this example study, the key parameters are:

- the maximum distance that the Romeo is tethered ${ }^{i i i}$ to the FFH

- the mix of sensors that the Romeo employs to detect, localise and classify contacts

- the search tactic that the Romeo employs to detect, localise and classify contacts 
Chandran et al., The use of human-in-the-loop and constructive simulation to support operations research into MH-60R tactics development

These parameters are then varied in CHOPPA, in addition to certain critical scenario characteristics such as the location and disposition of cargo ships, oil tankers, pleasure craft and fast-boat threats, which are randomised. In order to obtain statistically robust results, hundreds of runs of each valid parameter combination were conducted, ensuring that at least $95 \%$ of all outcomes were bounded within confidence intervals.

\subsection{Post Processing/Transmission of Outputs}

Following the completion of the simulation runs in CHOPPA, the outcomes are processed to examine the impact of changes to the key parameters on MOEs. Figure 5 and Figure 6 show fictitious representations for two of these outcomes.

Figure 5 shows that for all sensor mixes, the lowest number of fast-boat penetrations of the VA occur for low and extreme tether distances. Further, employing sensor mix 4 results in the fewest fast-boat penetrations of the VA across all tether distances.

Figure 6 shows that employing sensor mix 4 results in the provision of the greatest early warning to the FFH, meaning that the FFH has the best opportunity to defeat the threat.

These outcomes, in combination with the study approach, scenario, models, data and assumptions are reported to stakeholders for assessment and refinement if necessary. The recommendations provided have informed operators about how to improve the effectiveness of their mission, and will allow them to test these proposed approaches in an operational context

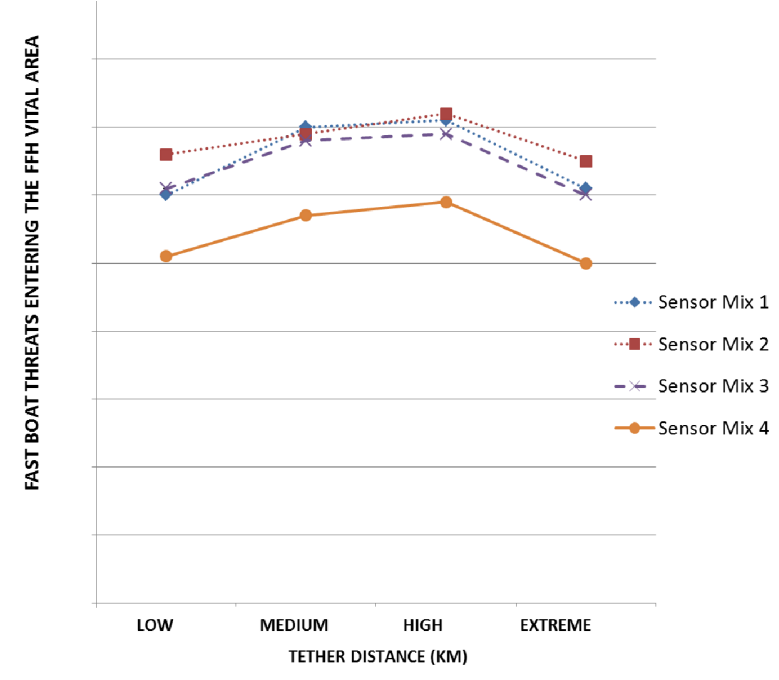

Figure 5. The number of fast-boat threats that penetrate the FFH VA based on sensor mix and tether distance

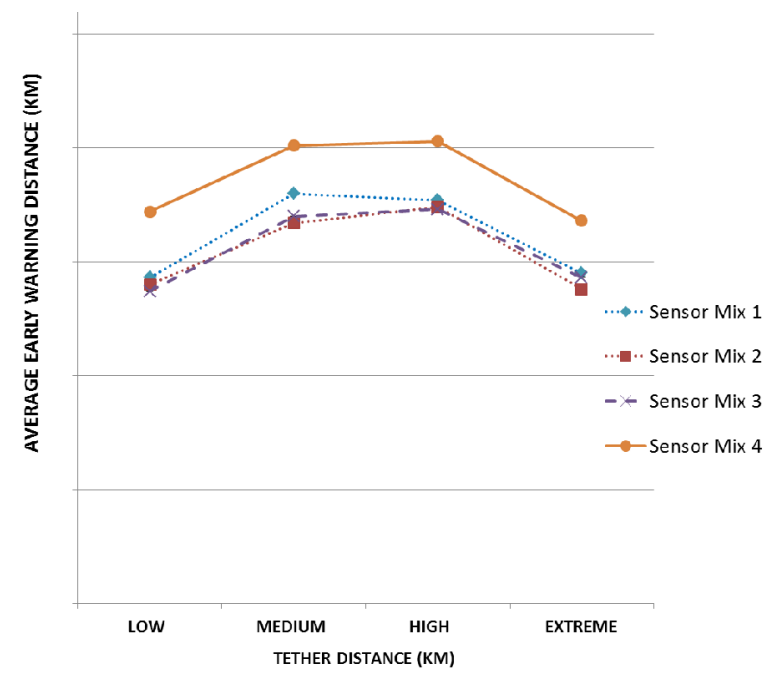

Figure 6. The average early warning distance based on sensor mix and tether distance

\section{FUTURE DEVELOPMENT AND CONCLUDING REMARKS}

The complementary use of SHARP and CHOPPA discussed in the paper highlights the value of the approach to engage and inform stakeholders to improve the conduct of military operations. As further studies are identified by the RAN, more work will be required to develop models of different fidelity, obtain data and integrate capability into SHARP and CHOPPA to further explore and analyse the Romeo problem space in a complex environment.

The capabilities that have been developed and used have had a considerable impact on the RAN, by contributing to the reduction in costs, increased effectiveness of RAN capability and improved co-ordination with Australia's allies. 
Chandran et al., The use of human-in-the-loop and constructive simulation to support operations research into MH-60R tactics development

\section{ACKNOWLEDGEMENTS}

The authors would like to acknowledge the input of the Australian Maritime Warfare Centre and the Fleet Air Arm for providing input and guidance to the study requirements. In addition, Advanced VTOL

Technologies and CAE have provided support in developing the SHARP and CHOPPA capabilities. These capabilities are funded by the RAN.

\section{REFERENCES}

Cullen, J. and Bryman, A. (1988). The knowledge acquisition bottleneck: time for reassessment? Expert Syst. Vol 5 No 3 (August 1988) pp 216-225

Macal, C. and North, M. (2010) Tutorial on agent-based modelling and simulation. Journal of Simulation 4: 151-162.

Burton, R. and Obel B. (2011) Computational modeling for what-is, what-might-be, and what-should-be studies-And triangulation. Organization science 22: 1195-1202

Bennett, K., Josefsson, T., Goss, S., Cross, M., Waugh, S. and Truong, T. (2001). An Application of DSTO’s Battlemodel using Agents and Humans-in-the-loop. Presented at SimtecT.

Iob, M., Chandran, A., Bramley, K., Dart, P., Lawrie, G., and Tidhar, G. (2004). Experimentation, Operations Research and the AEW\&C Armchair Warrior Exercises. Presented at the Defence Experimentation Symposium.

Tidhar, G., Heinze, C. and Selvestrel, M. (1998). Flying Together: Modelling Air Mission Teams. Applied Intelligence 8 (3), pp. 195-218.

Chandran, A., Ibal, G. and Tu, Z. (2007). Developing ARH Troop Tactics Using Operations Research Constructive Simulation. Presented at MODSIM 2007.

Homeland Security (2003). USS Cole bombing Suicide Attack. http://www.globalsecurity.org/security/profiles/uss_cole_bombing.htm

TTCP (2006). Guide for Understanding and Implementing Defense Experimentation GUIDEx pp 175-176

\footnotetext{
i The 'vital area' is a predetermined no-go region of a given radius around the FFH.

ii The majority of information is obtained from other DST Group divisions and the Australian Maritime Warfare Centre.

iii The 'tether' distance is the maximum separation distance between the Romeo and the FFH.
} 